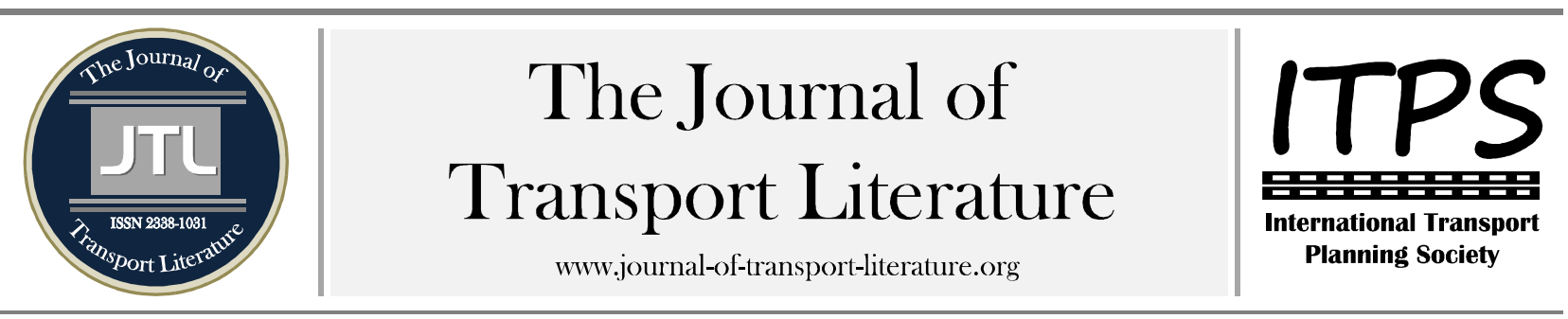

\title{
Avaliação de desempenho pelo MEPDG: o efeito da variação de espessuras de pavimentos flexíveis
}

Nayara Donelli Pelisson ${ }^{1}$; José Leomar Fernandes Júnior²; Carlos Alberto P. Silva Júnior ${ }^{1}$; Heliana Barbosa Fontenele ${ }^{1,+}$

${ }^{1}$ Londrina State University, Londrina, Brazil

2 São Paulo University, São Carlos, Brazil

\section{Article Info}

Palavras-chave:

MEPDG

pavimentos flexíveis

dimensionamento

desempenho

Submitted 25 Nov 2013;

received in revised form 28 Aug 2014;

accepted 8 Oct 2014.

Licensed under

Creative Commons

CC-BY 3.0 BR.

\section{Resumo}

O dimensionamento de pavimentos tem sido realizado em bases empíricas, que apresentam limitações, pois seus resultados são válidos apenas para os mesmos, ou muito similares, fatores intervenientes que prevaleciam quando do seu desenvolvimento. Dessa forma, a AASHTO propôs um novo método para o projeto de pavimentos, cuja base é empírico-mecanística. Tal método, conhecido inicialmente como AASHTO 2002, utiliza para o dimensionamento e previsão do desempenho os espectros de carga por eixo dos veículos comerciais de carga, além de informações detalhadas sobre o clima e materiais das camadas. Neste trabalho foi realizada uma análise de sensibilidade para investigar o efeito que a variação das espessuras do revestimento, da base e da sub-base acarreta nas respostas estruturais do pavimento flexível, bem como na previsão do seu desempenho. Foram feitas simulações com o programa computacional MEPDG utilizando-se espectros de carga desenvolvidos por Fontenele (2011). Os resultados quantificaram a magnitude dos efeitos causados pelos fatores selecionados e suas interações sobre o desempenho final do pavimento; podendo, portanto, contribuir para melhorias no dimensionamento de pavimentos.

+ Corresponding author. Universidade Estadual de Londrina, Centro de Tecnologia e Urbanismo, Departamento de Construção Civil. Rodovia Celso Garcia Cid Pr 445 Km 380 - Campus Universitário. Jardim Universitário. Londrina - PR. Brasil. CEP: 86051-980.

E-mail address: heliana@uel.br.

\section{Introdução}

0 presente trabalho objetiva investigar o efeito que a variação das espessuras do revestimento, da base e da sub-base acarreta nas respostas estruturais do pavimento flexível, bem como no seu desempenho durante a vida útil.

Para desenvolver a análise de sensibilidade proposta, foram feitas simulações com o programa computacional MEPDG (Mechanistic-Empirical Pavement Design Guide), utilizando-se espectros de carga por eixo desenvolvidos por Fontenele (2011). A variação utilizada na pesquisa foi de apenas um parâmetro de projeto por vez (espessura das camadas), enquanto todos os outros permaneceram constantes.

Tendo em vista que o dimensionamento de pavimentos a partir de métodos empírico-mecanísticos tem se tornado cada vez mais promissor, a pesquisa contribuirá para o estudo dos parâmetros de maior (ou menor) sensibilidade no dimensionamento de pavimentos flexíveis para as condições de tráfego, materiais e climas brasileiros.

Neste tocante, será possível verificar a consistência das informações que o programa MEPDG fornece para auxiliar o projetista na busca de uma alternativa de dimensionamento. Desta forma, avaliar a sensibilidade do método a ser utilizado, antes de usá-lo na prática, contribuirá para o aperfeiçoamento do dimensionamento de pavimentos rodoviários e para a redução dos insucessos caracterizados pela deterioração precoce dos mesmos.

0 artigo é estruturado em quatro seções principais. A primeira delas se refere à revisão bibliográfica do trabalho, com uma abordagem sucinta do método empírico-mecanístico e do software MEPDG. A segunda seção é voltada ao método empregado na pesquisa detalhando as etapas e condições consideradas no desenvolvimento do trabalho. Na terceira seção são apresentados os resultados e as discussões relativas ao efeito das variações das espessuras. Por fim, a quarta seção é voltada à conclusão, sendo apontadas as principais constatações, a partir dos resultados obtidos.

\section{Revisão bibliográfica (background)}

Nas últimas décadas vários estudos buscaram representar com maior proximidade a realidade de campo às condições impostas pelo tráfego e seus efeitos no pavimento para alcançar estruturas com comportamento adequado e previsível perante as solicitações esperadas durante o período de projeto.

Assim, os métodos empírico-mecanísticos tem se tornado cada vez difundidos por serem descritos como aqueles que mais se aproximam do comportamento em campo. Com o auxílio computacional, as respostas de uma estrutura prédimensionada são determinadas e são calculados os danos acumulados durante a sua vida útil. Isso permite comparar o comportamento do pavimento com os critérios de desempenho estabelecidos para a vida em serviço e caso não sejam 
obedecidos, é necessário variar os parâmetros do projeto definido até que a condição ideal seja obtida (Fernandes Jr., Pais e Pereira, 2007; Fontenele, 2011).

A partir de uma pesquisa do National Cooperative Highway Research Program (NCHRP) foi desenvolvido o projeto 1-37 A. No projeto foi apresentado um novo método, de base empírico-mecanística, para o dimensionamento de pavimentos novos e reabilitados. 0 AASHTO 2002, como conhecido inicialmente, continha um programa computacional conhecido como MEPDG. No novo método a forma de caracterização do tráfego foi modificada, sendo feita a partir dos espectros de carga por eixo (Axle Load Spectra).

Segundo Haider e Harichandran (2007), os espectros de carga por eixo consistem em informações de carga por eixo e de suas repetições para várias configurações (eixos simples, tandem duplos e triplos), sendo usados para criar distribuições que representem as cargas dos eixos e as variações a elas associadas.

0 programa MEPDG analisa o desempenho da estrutura de um pavimento mediante critérios pré-estabelecidos. 0 software utiliza uma aproximação hierárquica na incorporação das variáveis de entrada, em função da importância do projeto e da disponibilidade dos dados. Tal aproximação se refere aos dados de entrada de tráfego, materiais e meio ambiente. A partir dos resultados obtidos no MEPDG, é possível conduzir uma análise de sensibilidade para verificar os efeitos da variação dos parâmetros de projeto no comportamento dos pavimentos ao longo de sua vida em serviço.

De acordo com Schwartz et al. (2013), uma análise de sensibilidade é a distribuição da variabilidade dos dados de saída de um modelo para os seus diversos dados de entrada. A análise de sensibilidade por eles conduzida em pavimentos flexíveis sob cinco condições climáticas e três níveis de tráfego demonstrou que um dos dados de projeto mais consistentes nas categorias de sensibilidade é a espessura do revestimento asfáltico. As trincas longitudinais e por fadiga também apresentaram sensibilidade com relação à espessura da base.

Shahji (2006) avaliou a sensibilidade de pavimentos flexíveis e rígidos pelo MEPDG e concluiu que há maior influência da variação das espessuras do revestimento para a ocorrência dos defeitos. O autor afirma que as trincas do tipo up-down são menos sensíveis aos parâmetros tráfego, espessuras e Módulos de Elasticidade, mas são muito sensíveis à variação da espessura do revestimento asfáltico. Foi observado também que as trincas do tipo bottom-up são mais influenciadas pelos parâmetros de tráfego (VDMA) e espessura do revestimento asfáltico e têm pouco efeito pelos outros parâmetros de projeto, como espessura da sub-base e Módulo de Elasticidade do subleito.

Os resultados da pesquisa de Shahji (2006) levaram à conclusão que as mudanças nas propriedades da base, sub-base e subleito não têm muito impacto na deformação do pavimento, o que o autor salienta como "não muito realista". Com relação à deformação permanente total do pavimento, foi percebido que houve maior influência devido ao tráfego e à espessura da camada asfáltica. 0 autor concluiu, por fim, que o desempenho do pavimento depende da combinação de espessuras de camada asfáltica e do módulo de subleito usados para o projeto.

Ainda, segundo Derong et al. (2014), estudos anteriores fizeram o uso de várias abordagens no que concerne à análise de sensibilidade. Como exemplos, tem-se os estudos conduzidos por Smith e Diefenderfer (2010), por Haider et al. (2011), por Romanoschi et al. (2011), e por Bayomy et al. (2012) que utilizaram os defeitos deformações permanentes, trincas por fadiga e o Índice de Irregularidade Internacional (IRI) como indicadores de sensibilidade.

\section{Método}

0 procedimento desenvolvido nesta pesquisa teve início com a determinação dos parâmetros de projeto a serem variados. A análise de sensibilidade consistiu na variação de apenas um parâmetro de projeto por vez, enquanto todos os outros permaneceram constantes.

Definiu-se para a análise a variação das espessuras das camadas de revestimento, base e sub-base, como forma de investigar o seu efeito nas respostas estruturais e, consequentemente, no desempenho do pavimento. Posteriormente foram definidos os dados de entrada no programa computacional MEPDG, bem como a determinação dos critérios de desempenho da estrutura analisada, seguindo as diretrizes do Guia de Dimensionamento da AASHTO 2002.

As subsessões que seguem apresentam de forma detalhada os dados de entrada usados no programa MEPDG, sendo eles referentes à estrutura e propriedade dos materiais das camadas, ao tráfego, clima e critérios de projeto.

\subsection{Estrutura}

As características da estrutura de pavimento utilizadas nas simulações desta pesquisa são apresentadas a seguir. Na Tabela 1 estão os dados relativos às espessuras, módulo de elasticidade e coeficiente de Poisson.

Tabela 1 - Espessuras das camadas, Módulo de Elasticidade e Coeficiente de Poisson

\begin{tabular}{cccc}
\hline Camada & $\begin{array}{c}\text { Espessura } \\
(\mathbf{c m})\end{array}$ & $\begin{array}{c}\text { Módulo de Elasticidade } \\
(\text { MPa) }\end{array}$ & $\begin{array}{c}\text { Coeficiente } \\
\text { de Poisson }\end{array}$ \\
\hline 1 & 7,$5 ; 10,0 ; 12,5 ; 15,0$ & 4.000 & 0,30 \\
2 & 15,$0 ; 17,5 ; 20,0 ; 22,5$ & 400 & 0,40 \\
3 & 20,$0 ; 22,5 ; 25,0 ; 27,5$ & 200 & 0,40 \\
Subleito & $\infty$ & 70 & 0,45 \\
\hline
\end{tabular}

Para o revestimento asfáltico (camada 1) foi considerada distribuição granulométrica conforme a faixa C do DNIT, CAP60-70, teor igual a 6\% e volume de vazios da mistura igual a 4\%. A base de brita graduada simples (camada 2) possuía distribuição granulométrica de acordo com a faixa C do DER-SP. Para a sub-base (camada 3) considerou-se solo A-2-6 e o subleito (camada 4) foi de solo tipo A-7-6.Para todas as camadas foi adotado o nível 3 do MEPDG (de menor precisão), sendo utilizados dados médios da região de coleta. Para parâmetros não especificados acima, foram utilizados dados padrões do programa.

\subsubsection{Tráfego}

Os dados de tráfego foram considerados como nível 1 (maior precisão). Foram inseridos no programa a distribuição de veículos por classe do volume diário médio anual de tráfego (VDMA), o fator de crescimento linear, espectros de carga por eixo desenvolvidos por Fontenele (2011) e o número de eixos por caminhão. 
Os espectros de carga correspondiam aos dados do posto de pesagem do km 28 da Rodovia dos Imigrantes do ano de 2008. O VDMA no ano base de 2009, obtido no site do DER-SP, foi de 10.993 veículos/dia. A taxa adotada para o crescimento do tráfego foi de $4 \%$ ao ano e a pressão de enchimento dos pneus foi de $827 \mathrm{kPa}$ (120 psi).

\subsection{Clima}

Foi considerada a condição climática de Orlando (Flórida- EUA), em função da similaridade dos índices pluviométricos da região onde se encontra a Rodovia dos Imigrantes. A profundidade do lençol freático foi de $10 \mathrm{~m}$.

\subsection{Critérios de desempenho}

O nível de confiança considerado para todos os critérios foi de 90\%, conforme é proposto pela NCHRP (2004). Os critérios de desempenho foram: IRIinicial de aproximadamente $1 \mathrm{~m} / \mathrm{km}$; IRIfinal igual a $4 \mathrm{~m} / \mathrm{km}$; Trincas longitudinais updown de $189 \mathrm{~m} / \mathrm{km}$; Trincas por fadiga classe 3 bottom-up igual a 25\%; Afundamento nas trilhas de rodas no CBUQ igual a $6,35 \mathrm{~mm}$ e Afundamento nas trilhas de roda do pavimento todo igual a 19,5 mm.

\section{Resultados e discussões}

A partir das curvas de desempenho geradas pelo MEPDG foi possível desenvolver uma análise de sensibilidade alterando a espessura de cada uma das camadas por vez, mantendo as outras constantes. Os valores de referência, que se mantiveram fixos quando da variação de cada uma das espessuras das camadas, foram de $12,5 \mathrm{~cm}$ para o revestimento, $20 \mathrm{~cm}$ para a base e $25 \mathrm{~cm}$ para a sub-base. Logo, ao se variar a espessura do revestimento, as espessuras de base e sub-base ficaram inalteradas, conforme os valores citados.

Nas Figuras 1, 2 e 3 são apresentados os gráficos gerados a partir dos valores previstos para o $20^{\circ}$ ano de projeto. Pode ser observado que todos os valores apresentados ultrapassaram o limite de projeto, exceto os relativos à IRI. A estrutura não foi alterada de forma a buscar a adequação em termos de critérios máximos de projeto, tendo em vista que o objetivo desta pesquisa era apenas de verificar a sensibilidade à variação dos parâmetros de projeto.

Inicialmente, a análise foi feita para a variação da espessura da Camada 1 (revestimento asfáltico) de 7,5 a 15,0 cm. Os resultados quanto a essa variação são apresentados na Figura 1.
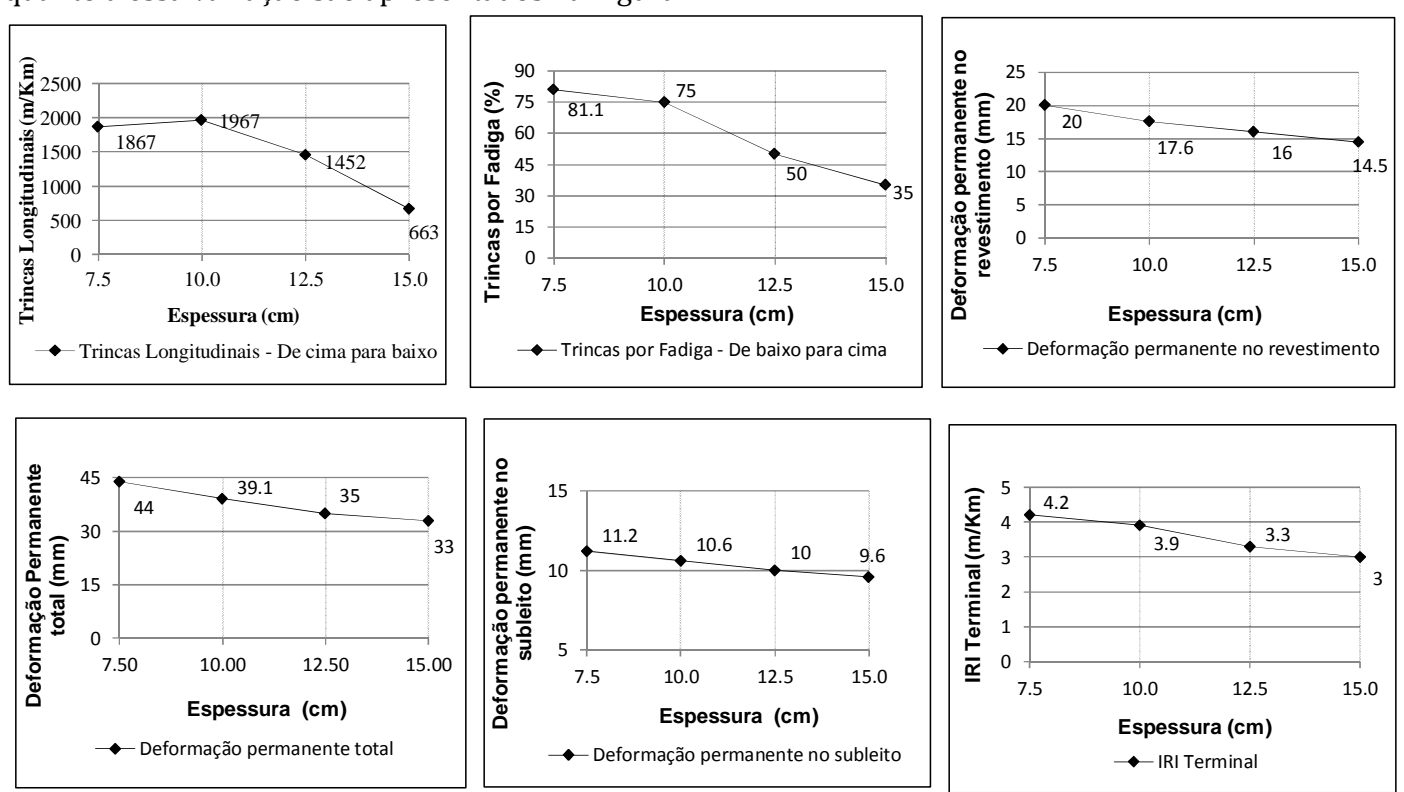

Figura 1 - Variação das medidas relativas às deteriorações analisadas para a variação da espessura do revestimento Fonte: Dos autores. "Com cálculos próprios"

A partir da Figura 1 observa-se que à medida que se aumenta a espessura da camada, ocorre uma redução dos defeitos analisados, revelando que os defeitos são sensíveis à variação de espessura da camada. Para as trincas longitudinais (de cima para baixo) é possível verificar uma variação de 66\% do valor mínimo em relação ao máximo. As trincas por fadiga (de baixo para cima) sofreram uma redução igual a $57 \%$. As deformações permanentes (apenas no revestimento) apresentaram uma redução de $27,5 \%$. Já com relação às deformações permanentes em todo o pavimento, é apresentada uma redução do defeito de $25,0 \%$. A deformação permanente apenas no subleito apresentou uma variação de 14,3\% entre os extremos. 0 IRI reduziu em $29 \%$.

A segunda análise foi feita para a variação da espessura da Camada 2 (base de brita graduada simples. Os resultados são apresentados na Figura 2. Observando a Figura 2 é possível verificar que à medida que a espessura da base aumenta ocorre uma redução do defeito do tipo trincas longitudinais (de cima para baixo). A variação de cerca de $34 \%$ do valor mínimo em relação ao máximo revela que o defeito é sensível à variação de espessura da camada. As trincas por fadiga (de baixo para cima) também sofrem uma redução de valores, mas de cerca de 15\%, o que indica que o defeito não é tão sensível quanto às trincas longitudinais à variação de espessura da base.

As deformações permanentes apenas no revestimento tem uma redução com relação ao máximo de 3,75\%, assim percebe-se que o defeito é pouco sensível a tal variação. Com relação às deformações permanentes em todo o pavimento, é apresentada uma redução do defeito de 2,8\%, o que expressa quase nenhuma sensibilidade do defeito em função da variação da espessura da camada. 0 mesmo ocorre com a deformação apenas no subleito, havendo uma variação de $11,8 \%$ entre os extremos. O IRI apresentou redução de cerca de $6 \%$ indicando que a irregularidade longitudinal também não é tão sensível à variação da espessura da camada. 

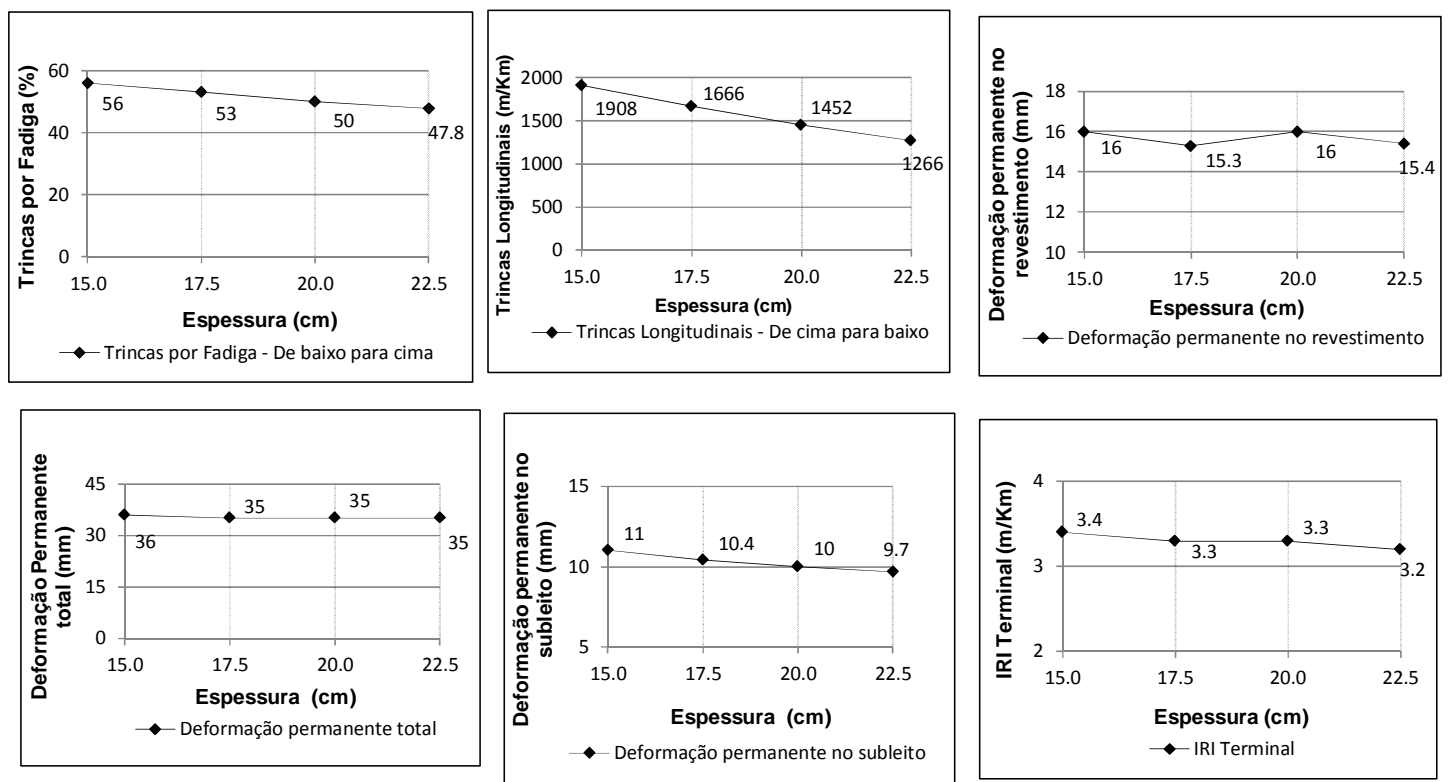

Figura 2 - Variação das medidas relativas às deteriorações analisadas para a variação da espessura da base Fonte: Dos autores. "Com cálculos próprios"

A última análise foi com a variação da espessura da Camada 3 (sub-base). Os resultados são apresentados na Figura 3. A partir da observação da figura é possível verificar que ocorre um aumento na progressão do defeito trincas longitudinais (de cima para baixo) à medida que a espessura da camada aumenta. Foi verificado que houve uma variação de cerca de $5 \%$ do valor mínimo em relação ao máximo, revelando que o defeito não é tão sensível à variação de espessura da camada. As trincas por fadiga (de baixo para cima) sofrem uma redução de valores com o aumento da espessura da camada. A redução corresponde a uma porcentagem de cerca de 2,7\%, o que indica que o defeito é pouco sensível à variação de espessura da sub-base.
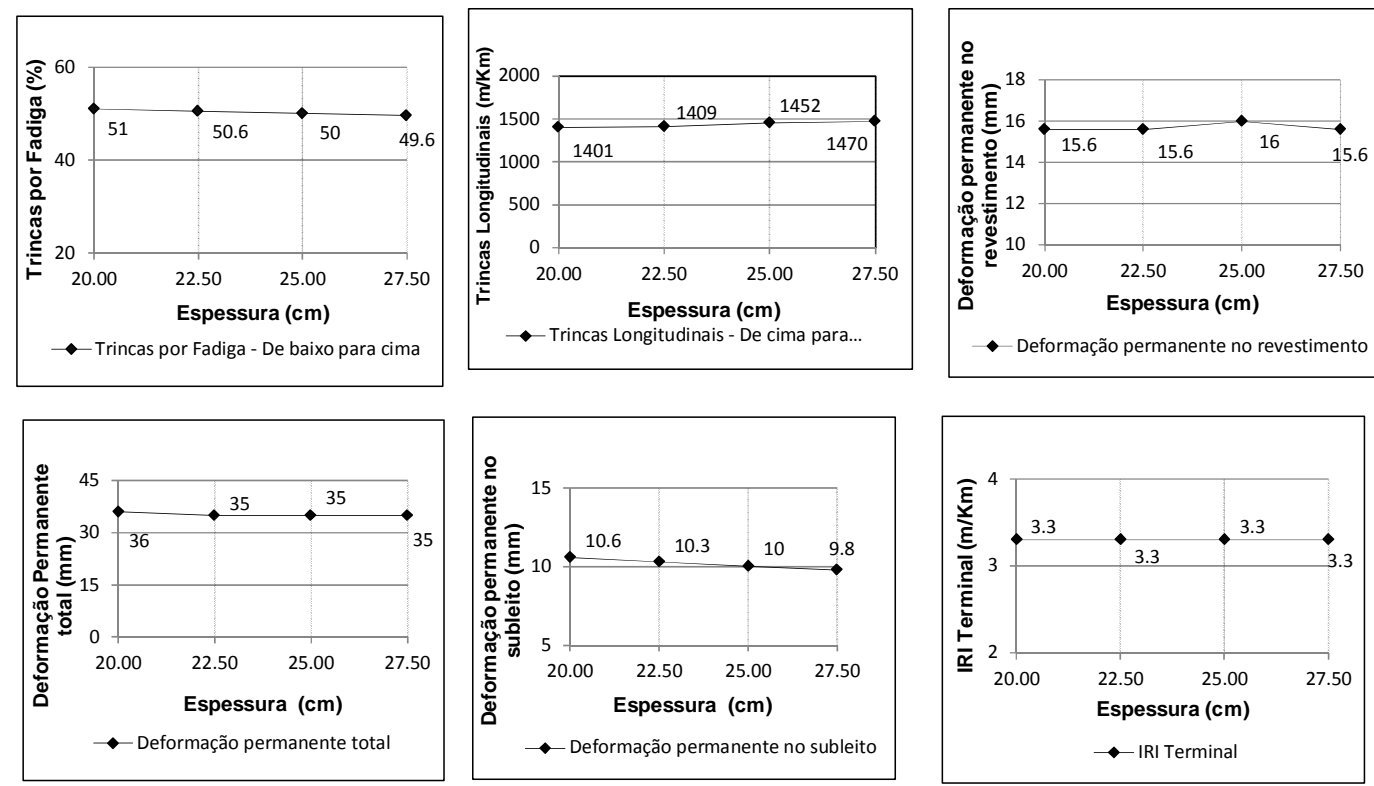

Figura 3 - Variação das medidas relativas às deteriorações analisadas para a variação da espessura da sub-base Fonte: Dos autores. "Com cálculos próprios"

As deformações permanentes apenas no revestimento são pouco sensíveis à modificação da espessura da camada, visto a pequena diferença entre os valores obtidos. Na deformação permanente total, os valores permanecem praticamente inalterados o que expressa nenhuma sensibilidade do defeito em função da variação da espessura da camada. A deformação somente no subleito sofre uma redução com o aumento da espessura da camada, havendo uma variação considerável de $11,8 \%$ entre os valores extremos. O IRI apresentou valor constante para todas as espessuras de sub-base demonstrando que não é sensível à variação da espessura da camada.

\section{Conclusão}

Neste trabalho foi desenvolvida uma análise de sensibilidade com o programa MEPDG a partir da variação das espessuras de um pavimento flexível. As informações de tráfego, estrutura, clima e demais características do pavimento foram baseadas no trabalho de Fontenele (2011).

A investigação do efeito da variação de certos parâmetros usados em projeto de pavimentos se torna importante porque pode levar à obtenção de informações que contribuam para o Sistema de Gerência de Pavimentos (SGP) a partir do aperfeiçoamento no dimensionamento de pavimentos e da diminuição dos insucessos caracterizados pela deterioração precoce de pavimentos. 
A análise realizada possibilitou verificar que a ocorrência dos defeitos trincas longitudinais e trincas por fadiga são bem mais sensíveis quando se varia a espessura do revestimento asfáltico. Também houve certa sensibilidade quando a espessura da base foi variada, entretanto, em menor magnitude, havendo ainda uma diminuição de sensibilidade do defeito trincas por fadiga quando comparado às trincas longitudinais. Vale ressaltar que tais defeitos pouco foram influenciados com a variação da espessura da sub-base.

A deformação permanente no revestimento apresentou maior sensibilidade com a variação da espessura do revestimento e, quase nenhuma, com as da base e sub-base. Tal situação também ocorreu para a deformação total, porém apresentando uma menor sensibilidade à variação da espessura do revestimento quando comparada à deformação apenas no revestimento. Para o IRI, foi constatada uma maior sensibilidade com a variação da espessura do revestimento, sendo muito pouco influenciado pelas variações relacionadas à base e nenhuma pela da sub-base.

Diante do exposto, quanto à variação de espessuras constata-se que, independentemente das conformações de base e sub-base, a espessura do revestimento foi o parâmetro que mostrou ter o maior efeito, principalmente no que se refere ao defeito trincas. Confirmam-se, assim, as evidências de estudos anteriores, inclusive os que levaram à adoção, por parte da AASHTO (1986), de espessura mínima de $12,5 \mathrm{~cm}$ para o revestimento asfáltico de rodovias com tráfego médio, correspondente a um número de aplicações do eixo-padrão relativamente baixo (maior que 5 x 106).

No Brasil, todas as evidências relacionadas à importância da espessura da camada de revestimento asfáltico têm sido ignoradas, com adoção de valores que, se representam alguma economia no custo de construção, resultam em custos altíssimos de manutenção e reabilitação e, mais ainda, de operação de veículos ao longo da vida em serviço.

Para obter uma análise mais aprofundada, sugere-se que sejam consideradas, futuramente, as variações de outros parâmetros de projeto como os módulos de elasticidade, taxas de crescimento do tráfego e tipos de ligantes asfálticos. 0 uso de maior quantidade de dados apoiados em resultados laboratoriais é outra consideração interessante. Ainda uma análise estatística mais detalhada com uma matriz fatorial mais abrangente e conciliada a uma análise de sensibilidade global, virá futuramente, a contribuir para o aperfeiçoamento da pesquisa.

Além disso, pode ser considerada a comparação dos resultados obtidos pela metodologia aqui adotada com a empírica, bem como comparar o produto alcançado a partir dos modelos utilizados pelo MEPDG com outros modelos de previsão de desempenho como, por exemplo, os desenvolvidos por Soncim e Fernandes Júnior (2014).

Agradecimentos

À Fundação Araucária pelo auxílio financeiro à pesquisa de Protocolo nº 24685 - Chamada 14/2011.

\section{References}

American Association of State Highway and Transportation Officials - AASHTO (1986). Guide for Design of Pavement Structures. Washington, D. C.

Bayomy, F., El-Badawy, S., \& Awed, A. (2012). Traffic characterization. Chapter 6, Implementation of the MEPDG for flexible pavement in Idaho, Idaho Transportation Department Rep. 193, Univ. of Idaho, Moscow, ID, 123-174.

Derong, Turochy, E. R., \& Timm, H. D. (2014). Sensitivity of Flexible Pavement Thickness to Traffic Factors in Mechanistic-Empirical Pavement Design. Journal of Transportation Engineering.

Fernandes Júnior, J. L., Pais, J. C., \& Pereira, P. A. A. (2007). Avaliação dos efeitos das solicitações do tráfego sobre o desempenho de pavimentos flexíveis portugueses e brasileiros. Revista Pavimentação, Ano II, 5, 28-38. Rio de Janeiro: ABPv.

Fontenele, H. B. (2011). Representação do Tráfego de Veículos Rodoviários de Carga através de Espectros de Carga por Eixo e seu Efeito no Desempenho dos Pavimentos. Tese (Doutorado) -Escola de Engenharia de São Carlos da Universidade de São Paulo.

Haider, S. W., \& Harichandran, R. S. (2007). Relating Axle Load Spectra to Truck Gross Vehicle Weights and Volumes. Journal of Transportation Engineering, 133 (12), 696-705.

Haider, S. W., Buch, N., \& Chatti, K. (2011). Development of traffic inputs for the Mechanistic-Empirical Pavement Design Guide in the state of Michigan. Transportation Research Record 2256, Transportation Research Board, Washington, DC, 179-190.

Research Board, Washington, DC, 179-190. National Cooperative Highway Research Program - NCHRP (2004) Guide for MechanisticEmpirical Design of New and Rehabilitated Pavement Structures. Final Report NCHRP 1-37 A. Champaign, Illinois.

Romanoschi, S. A., Momin, S., Bethu, S., \& Bendana, L. (2011). Development of traffic inputs for the new ME Pavement Design Guide: A case study. Proc., 90th Annual Meeting of the Transportation Research Board (CD-ROM), Washington, DC.

Schwartz, W. C., LI, R., Ceylan, H.; Kim, S., \& Gopalakrishnan, K. (2013). Global Sensitivity Analysis of Mechanistic-Empirical Performance Predictions for Flexible Pavements. Transportation Research Board. TRB 2013 Annual Meeting CD-ROM. Washington, D. C.

Shahji, S. (2006). Sensitivity Analysis of AASHTO's 2002 Flexible and Rigid Pavement Design Methods. 2006. Master of Science in the Department of Civil \& Environmental Engineering - College of Engineering and Computer Science - University of Central Florida. Orlando, Florida.

Smith, B. C., \& Diefenderfer, B. K. (2010). Analysis of Virginia-specific traffic data inputs for use with the Mechanistic-Empirical Pavement Design Guide. Rep. No. VTRC 10-R19, Virginia Transportation Research Council, Charlottesville, VA.

Soncim, S. P., \& Fernandes Jr, J. L. (2014) Modelo de previsión de la progresión del área de fisuras en pavimentos asfálticos. Journal of Transport Literature, 8(2), 221-243.

\section{Abstract}

The pavement design has been performed on empirical basis, which present limitations, because their results are only valid for the same, or very similar, intervening factors that prevailed at the time of its development. In this way, the AASHTO proposed a new method of pavements design, whose base is mechanistic-empirical. This method, which was initially known as AASHTO 2002, uses the axle load spectra of commercial vehicles for design and performance prediction, as well as detailed information about climate and layers materials. This work performed a sensitivity analysis to investigate the effect that thickness variation of bituminous and base layers can produce to the structural responses of flexible pavements and their effects on pavement performance. Thus, simulations were made with the computer program MEPDG using load spectra developed by Fontenele (2011). The results quantified the effects magnitude caused by the selected factors and their interaction on the final performance of pavements. Therefore, it can contribute to the improvement of pavement design.

Key words: MEPDG, flexible pavements, design, performance. 